\title{
Direct hydrogen gas generation by using InGaN epilayers as working electrodes
}

\author{
J. Li, J. Y. Lin, ${ }^{a)}$ and H. X. Jiang ${ }^{\text {b) }}$ \\ Nano-Tech Center, Texas Tech University, Lubbock, Texas 79409, USA
}

(Received 14 September 2008; accepted 4 October 2008; published online 24 October 2008)

\begin{abstract}
We report on the growth and exploitation of InGaN epilayers as a photoelectrochemical cell (PEC) material for direct generation of hydrogen by splitting water using photoelectrochemical hydrolysis. Under white light illumination, a drastic dependence of the photocurrent density on the In content was observed. Direct hydrogen gas generation by splitting water was accomplished using an $n$-type $\mathrm{In}_{x} \mathrm{Ga}_{1-x} \mathrm{~N}$ epilayer with a relatively high $\mathrm{In}$ content $(x \sim 0.4)$ as a working electrode. This demonstration of hydrogen generation by water splitting accomplished using InGaN based PEC is highly encouraging. (c) 2008 American Institute of Physics. [DOI: 10.1063/1.3006332]
\end{abstract}

Efficient and cost effective conversion of sunlight to hydrogen by splitting water is a major enabling technology for a viable hydrogen economy. ${ }^{1-3}$ The sunlight has sufficient energy to split water into hydrogen and oxygen if efficient light-harvesting systems can be developed and incorporated. The key is to find a light-harvesting system that can efficiently collect the photon energy and direct it toward the water splitting reaction. In photoelectrochemistry, the light harvesting system is a semiconductor immersed in aqueous solution. Most semiconductor materials have the potential to be constructed as photoelectrochemical cells (PEC) for directly generating hydrogen from water using solar energy, but they have unacceptably high corrosion rates. For example, GaInP, has shown promising efficiencies but suffers from poor stability. ${ }^{4,5}$

Currently, the most favored material for the photoanode in $\mathrm{PEC}$ is $\mathrm{TiO}_{2}$ due to its high corrosion resistance. ${ }^{5}$ However, $\mathrm{TiO}_{2}$ has an energy bandgap of about $3.2 \mathrm{eV}$ and can only be activated by light energy equal to or greater than 3.2 $\mathrm{eV}$. Such energy range is unfortunately present in less than $3 \%$ of the solar spectrum. This results in very low solar absorptivity in $\mathrm{TiO}_{2}$. Henceforth, they are intrinsically insufficient $(<2 \%)$ in systems for solar energy conversion such as in PEC. ${ }^{5}$ Considering the general science and engineering requirements, InGaN appears to be an ideal material for the development of next generation PEC because of their ability to provide tunable bandgap and bandgap engineering through alloying and nanostructure formation for highly efficient solar energy absorption. The energy gap of InGaN alloys can be tuned to cover the entire solar spectral region. ${ }^{6}$ Additionally, the smaller lattice constants or stronger bonding of In-N and $\mathrm{Ga}-\mathrm{N}$ of III nitrides compared with other semiconductors ( $\mathrm{GaP}$, for example) also implies higher corrosion resistance. Although III-nitride materials have been extensively studied for visible and ultraviolet light emitters and detectors during the past decade, very little work has been done with respect to their potential for electrolyzing water. ${ }^{7-11}$

Direct photoelectrolysis of water has recently been observed using GaN based PEC. ${ }^{8-11}$ Like $\mathrm{TiO}_{2}$, the energy gap of pure $\mathrm{GaN}(3.4 \mathrm{eV})$ is too high and does not match well with the solar spectrum and the necessary conditions for

\footnotetext{
a) Electronic mail: jingyu.lin@ttu.edu.

${ }^{b)}$ Electronic mail: hx.jiang @ttu.edu.
}

water splitting. The intrinsic properties of InGaN alloys, including their tunable energy gaps to cover the entire solar spectrum, expected high corrosion resistance, and their conduction and valance band edges can be made to straddle the $\mathrm{H}^{+} / \mathrm{H}_{2}$ and $\mathrm{O}_{2} / \mathrm{H}_{2} \mathrm{O}$ redox potentials, point to their great promises to be used as photoelectrodes for direct generation of hydrogen by splitting water using solar-powered photoelectrochemical hydrolysis. Here, we report on the demonstration of InGaN as a PEC material and show that the water splitting efficiency increases with an increase of the In content, (or equivalently as the bandgap of InGaN alloys is better matched with the solar spectrum).

The layer structure of InGaN epilayers used in water splitting experiment is shown in Fig. 1(a). $\operatorname{In}_{x} \mathrm{Ga}_{1-x} \mathrm{~N}$ epilayers of about $0.2 \mu \mathrm{m}$ thick were grown on top of a $1.5 \mu \mathrm{m}$ $\mathrm{GaN}$ epilayer by metal organic chemical vapor deposition. Prior to the GaN growth, a $25 \mathrm{~nm}$ thick GaN buffer layer was grown on $c$-plane sapphire at $550{ }^{\circ} \mathrm{C}$. Subsequent epilayer growth was carried out at $1050{ }^{\circ} \mathrm{C}$ for $\mathrm{GaN}$ and $710{ }^{\circ} \mathrm{C}$ for $\mathrm{In}_{x} \mathrm{Ga}_{1-x} \mathrm{~N}$. Trimethylgallium and trimethylindium were used as the precursors. Nitrogen and hydrogen were used as car- (a)

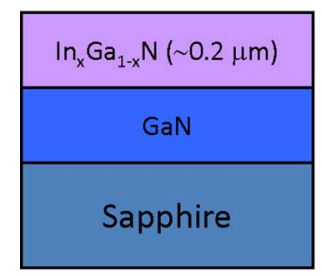

$500 \mu \mathrm{m}$

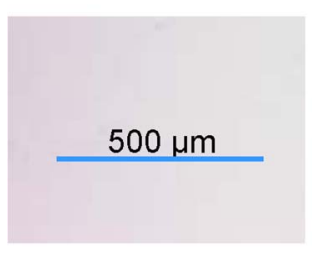

(b)

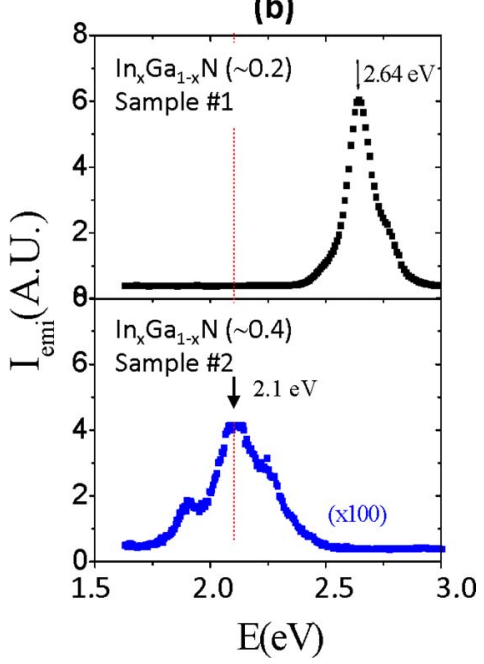

FIG. 1. (Color online) (a) Layer structure of InGaN epilayers used in water splitting experiment and InGaN epilayer surface probed by an optical microscope. (b) Room temperature photoluminescence emission spectra of two $n$ - $\operatorname{In}_{x} \mathrm{Ga}_{1-x} \mathrm{~N}$ epilayers (with $x \sim 0.2$ and 0.4 ) used in water splitting experiments. 


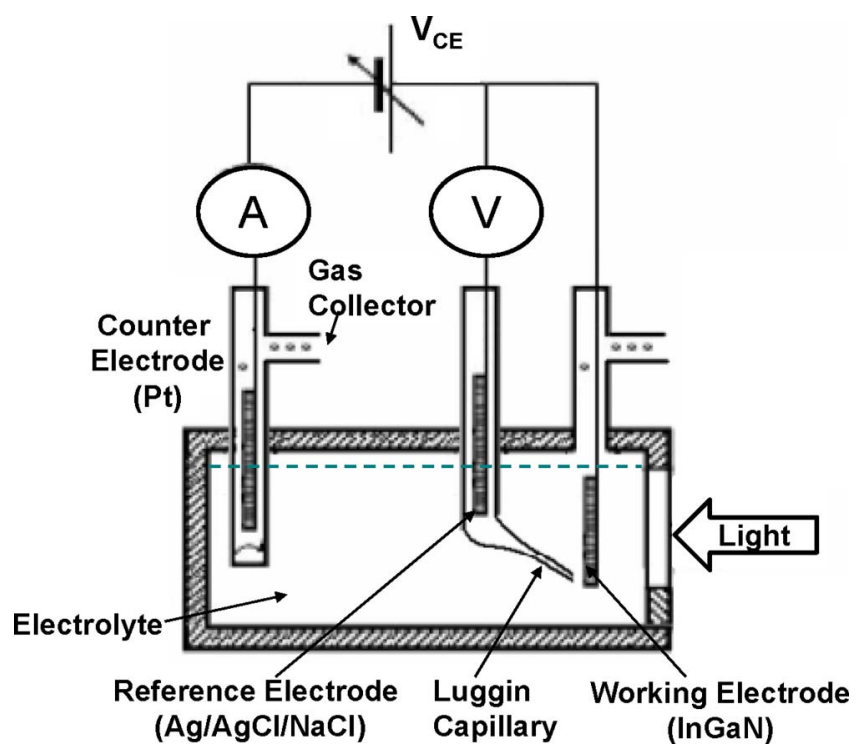

FIG. 2. (Color online) Schematic diagram of a photoelectrochemical cell (PEC) setup utilized to measure the water splitting efficiency of InGaN based PEC. Reactions take place on the surface of the working photoanode $(\mathrm{InGaN})$ and the cathode (Pt electrode) to produce oxygen and hydrogen, respectively.

rier gases for $\mathrm{InGaN}$ and $\mathrm{GaN}$, respectively. High purity ammonia was used as the active nitrogen source. To vary In content in InGaN, the TMIn flow rate was varied while other growth parameters were fixed. The $\operatorname{In}_{x} \mathrm{Ga}_{1-x} \mathrm{~N}$ epilayers were doped with $\mathrm{Si}$ at a flow rate of 0.25 SCCM (SCCM denotes cubic centimeter per minute at STP) of $10 \mathrm{ppm}$ silane. GaN and InGaN growth rates were $3.6 \mu \mathrm{m} / \mathrm{h}$ and $0.3 \mu \mathrm{m} / \mathrm{h}$, respectively. The typical room temperature electron concentration and mobility of InGaN alloys were $2 \times 10^{17} \mathrm{~cm}^{-3}$ and $160 \mathrm{~cm}^{2} / \mathrm{V} \mathrm{s}$, respectively, as determined by Hall effect measurements. As shown in Fig. 1(a), InGaN epilayers are crack free with a surface roughness of about $2 \mathrm{~nm}$ over a $10 \mu \mathrm{m}$ atomic force microscopy scanning area. Optical properties of InGaN alloys were characterized by photoluminescence (PL) measurements. Figure 1(b) shows the room temperature PL spectra of two $\operatorname{In}_{x} \mathrm{Ga}_{1-x} \mathrm{~N}$ epilayers used for water splitting experiment, where samples 1 and 2 have In contents of about 0.2 and 0.4 , respectively, as determined by $\mathrm{x}$-ray diffraction measurements ( $\theta$ and $2 \theta$ scans). Although the overall PL emission intensity of sample 2 is lower than that of sample 1 due its reduced crystalline quality with increasing In content, the bandgap of sample 2 with an emission peak around $2.1 \mathrm{eV}$ matches better with the solar spectrum.

The water splitting efficiencies of photocatalytic electrodes based upon InGaN materials were measured using a standard photoelectrochemical cell, as schematically illustrated in Fig. 2. The cell consists of a working electrode $(\mathrm{InGaN})$, a cathode (Pt counter electrode), and a sodiumchloride-saturated calomel silver-chloride electrode. The electrodes were immersed in the electrolyte solution. The potential difference (voltage) between the working and counterelectrodes $\left(V_{\mathrm{CE}}\right)$ was recorded to determine the applied bias and the photocurrent $\left(I_{\mathrm{p}}\right)$ was measured as a function of $V_{\mathrm{CE}}$.

We have obtained preliminary results by employing In$\mathrm{GaN}$ epilayers as working electrodes. As illustrated in Fig. 3, we have measured the photocurrent density as a function of

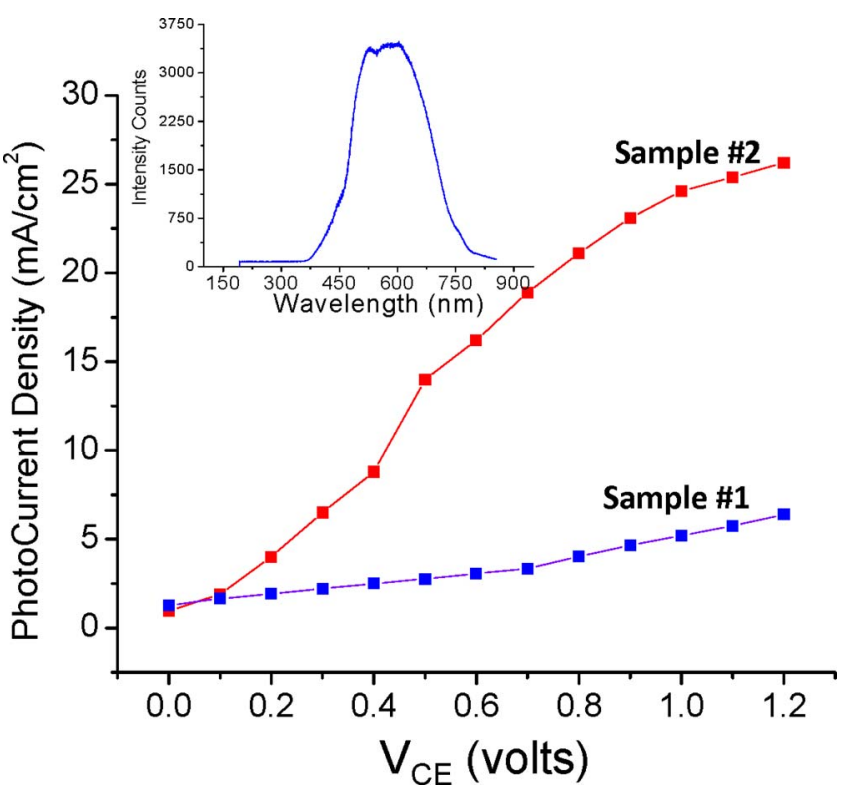

FIG. 3. (Color online) Photocurrent density vs $V_{\mathrm{CE}}$ (the bias voltage applied between the working and counter electrodes) of InGaN based PEC. The results were obtained in aqueous $1 \mathrm{~mol} / \mathrm{L} \mathrm{HCl}$ under static condition and white light illumination by using 2 different $n-\operatorname{In}_{x} \mathrm{Ga}_{1-x} \mathrm{~N}$ epilayers (with $x$ $=0.2$ and 0.4$)$ as working electrodes $\left(1 \times 1 \mathrm{~cm}^{2}\right) . \mathrm{H}_{2}$ generation is only visible when using sample 2 . The inset shows the emission spectrum of the white light source used for illumination.

$V_{\mathrm{CE}}$ by using samples 1 and 2 as working electrodes. Under white light illumination, a drastic dependence of the photocurrent density on the In content was observed. Sample 2 with lower PL emission energy (or higher In content) provides much higher photocurrent density. Since the overall material quality of sample $2(x \sim 0.4)$ is lower than that of sample $1(x \sim 0.2)$, the higher performance (higher photocurrent density) of sample 2 is solely due to the greater overlap of its energy bandgap with the solar spectrum. Furthermore, as shown in Fig. 4, when sample 2 was immersed in $1 \mathrm{M} / \mathrm{L}$ $\mathrm{HCl}, \mathrm{H}_{2}$ evolved vigorously from the $\mathrm{Pt}$ counter electrode. Our results suggest that the estimated In composition in In$\mathrm{GaN}$ as a photocatalytic electrode for optimal photoelectrolysis of water should be above 0.4. However, as we tune into the miscibility gap region in $\mathrm{InGaN}$ alloys with $\mathrm{InN}$ fraction between $45 \%-60 \%,{ }^{12}$ optimal effective composition will need to be determined by several complementary techniques due to the well known formation of In-rich nanoclusters (or quantum dots) ${ }^{13,14}$ in the middle composition range. On the

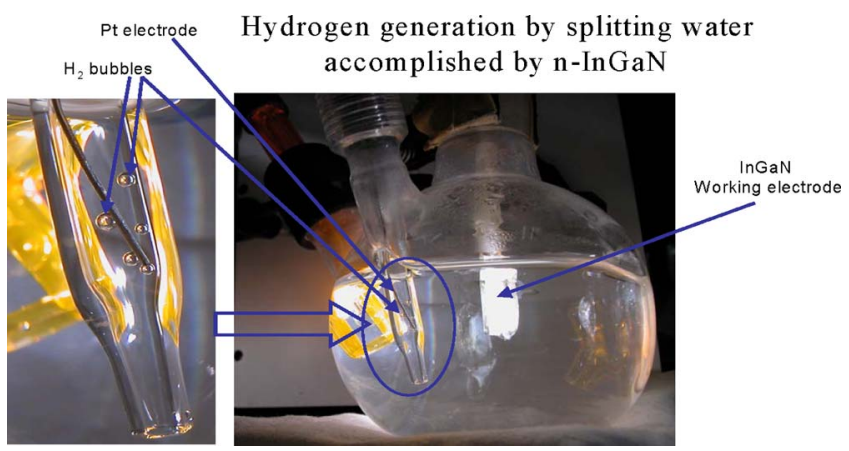

FIG. 4. (Color online) Photograph showing hydrogen gas $\left(\mathrm{H}_{2}\right.$ gas bubbles $)$ generation on the $\mathrm{Pt}$ counter electrode side through splitting water accomplished using an $n$-type $\mathrm{In}_{0.4} \mathrm{Ga}_{0.6} \mathrm{~N}$ epilayer as a working electrode under white light illumination. 
other hand, this spontaneous formation of nanoclusters in InGaN may be highly effective in increasing the water splitting efficiency. Furthermore, there is no visible physical degradation of the InGaN epilayers after several hours of measurements. InGaN based PEC cells are expected to provide superior corrosion resistance due to the small lattice constants and strong bonding between In/Ga and $\mathrm{N}$. However, prolonged measurements are needed to further study the corrosion effect and to verify this potential. Nevertheless, we believe that this demonstration of hydrogen generation by water splitting accomplished using $\mathrm{InGaN}$ based PEC is highly promising.

In summary, we have investigated the potential of InGaN epilayers as PEC materials. When employing $n$-type InGaN as a working electrode, a drastic dependence of the photocurrent density and hydrogen generation rate on the In content was observed. The sample with the lower PL emission energy (or higher In content) provides much higher photocurrent density and hydrogen generation rate. Compared with existing PEC technologies, III-nitrides have the potential to provide higher efficiency and durability and costeffective manufacturing and maintenance. III-nitrides also have high resistance to corrosion in aqueous water due to their small lattice constants. By varying In concentration, the band gap energies of InGaN alloys can be engineered to cover the entire solar spectrum and can be adjusted to opti- mally match with conditions for hydrogen generation by water splitting.

The InGaN epigrowth work is supported by NSF and the PEC characterization work is supported by AFOSR. J.Y.L. and H.X.J. gratefully acknowledge the support of the Linda Whitacre and Edward Whitacre Endowed Chair positions through the AT\&T Foundation and insightful discussions with Dr. Gernot S. Pomrenke of AFOSR.

${ }^{1}$ P. Weisz, Phys. Today 57(7), 47 (2004).

${ }^{2}$ G. W. Crabtree, M. S. Dresselhaus, and M. V. Buchanan, Phys. Today 57(12), 39 (2004).

${ }^{3}$ J. A. Turner, Science 285, 687 (1999).

${ }^{4}$ O. Khaselev and J. Turner, J. Electrochem. Soc. 145, 3335 (1998).

${ }^{5}$ A. J. Nozik and R. Memming, J. Phys. Chem. 100, 13061 (1996).

${ }^{6}$ J. Wu, W. Walukiewicz, K. M. Yu, W. Shan, J. W. Ager III, E. E. Haller, H. Lu, W. J. Schaff, W. K. Metzger, and S. Kurtz, J. Appl. Phys. 94, 6477 (2003).

${ }^{7}$ S. S. Kocha, M. W. Peterson, D. J. Arent, J. M. Redwing, M. A. Tischler, and J. Turner, J. Electrochem. Soc. 142, L238 (1995).

${ }^{8}$ K. Fujii, T. Karasawa, and K. Ohkawa, Jpn. J. Appl. Phys., Part 2 44, L543 (2005)

${ }^{9}$ K. Fujii and K. Ohkawa, Jpn. J. Appl. Phys., Part 2 44, L909 (2005).

${ }^{10}$ K. Fujii and K. Ohkawa, Phys. Status Solidi C 3, 2270 (2006).

${ }^{11}$ I. Waki, D. Cohen, R. Lal, U. Mishra, S. P. DenBaars, and S. Nakamura, Appl. Phys. Lett. 91, 093519 (2007).

${ }^{12}$ I. H. Ho and G. B. Stringfellow, Appl. Phys. Lett. 69, 2701 (1996).

${ }^{13}$ S. Nakamura, M. Senoh, N. Iwasa, and S. Nagahama, Jpn. J. Appl. Phys., Part 2 34, L797 (1995).

${ }^{14}$ S. Nakamura and G. Fasol, The Blue Laser Diode: GaN Based Light Emitters and Lasers (Springer, Berlin, 1997). 